\title{
'Dental Decisions' study launches
}

A study aiming to explore how patients and dentists make decisions about dental care for people living with dementia at all stages has launched.

The 'Dental Decisions' study is led by Andrew Geddis-Regan, a National Institute for Health Research (NIHR) Doctoral Research Fellow \& Hon. Special Care Dentist, and supervised by a multidisciplinary team, at Newcastle University.

This project, funded by NIHR as a Doctoral Fellowship, aims to work out how the decision-making process could be easier for patients living with dementia, carers and dentists.

The Alzheimer's Society Research Network, the North East Dementia Alliance and multiple people living with dementia and their carers have supported the design of the project and helped to determine its main focus and aims. These stakeholders raised concerns about living with pain which may not be identifiable; a paper was co-written with a carer helping to explore the published research about this problem. ${ }^{1}$ Patients also expressed concern about needing complicated treatment when they were too unwell to cope with it or when
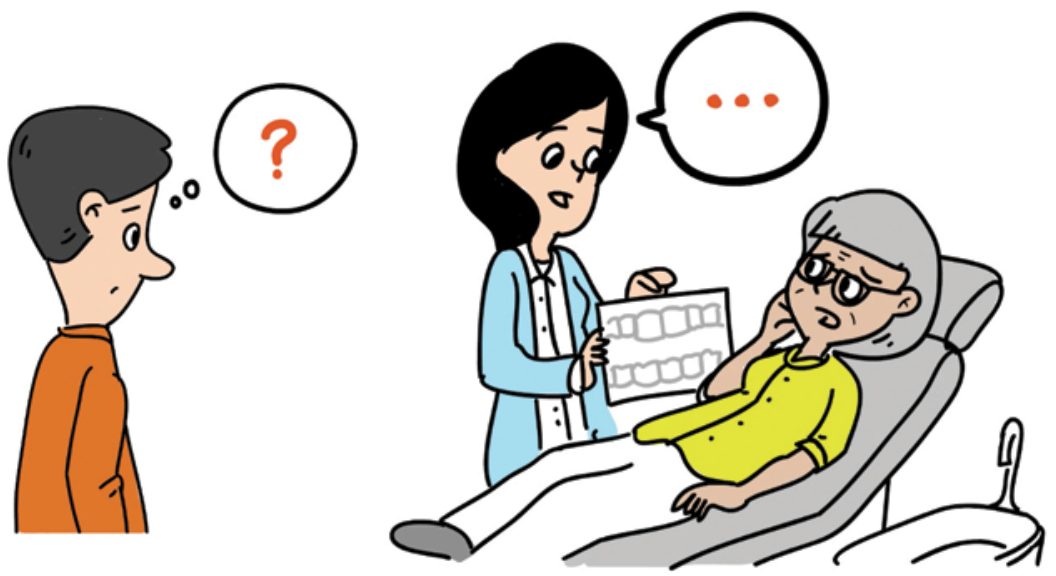

dental treatment might lead to more harm than benefit.

Dr Geddis-Regan said: 'The first stage of the research will involve observation of dental visits for people living with dementia in a number of community dental services. The study team are also interviewing dentists and patients or carers. The COVID-19 pandemic has meant the observation of visits will occur at a later stage, but the first interviews have begun in May 2020'.

Dentists can express an interest in taking part in an interview at https://research.ncl. ac.uk/dentaldecisions/.
Following this exploratory work, the project is working with the Lab4Living team at Sheffield Hallam University to design a practical solution to improve decisionmaking and dental care delivery for this patient group. Dentists, patients, carers and other professionals will be involved in this process and are invited to express their interest in contributing on the website.

\section{Reference}

1. Geddis-Regan A R, Stewart M, Wassall R R. Orofacial pain assessment and management for patients with dementia: a meta-ethnography. J Oral Rehabil 2019; 46: 189-199.

\section{Gum Health Day 2020 says 'No' to bleeding gums}

'Say NO to bleeding gums' was the slogan for Gum Health Day 2020, celebrated worldwide on 12 May.

Professor Andreas Stavropoulos, co-ordinator of Gum Health Day 2020, said that 'Gum Health Day 2020 aims to remind people that gum health is a key factor for general health and well-being throughout life, and that gum disease is an important public health issue as it is linked to very serious conditions'.

Gum diseases including gingivitis, periodontitis, and peri-implantitis are still poorly acknowledged by the public, even though scientific evidence shows that they may pose a threat to general health as they are associated with cardiovascular disease, diabetes, chronic kidney disease, rheumatoid arthritis, certain forms of cancer, pregnancy complications, erectile dysfunction, and other serious or chronic conditions.
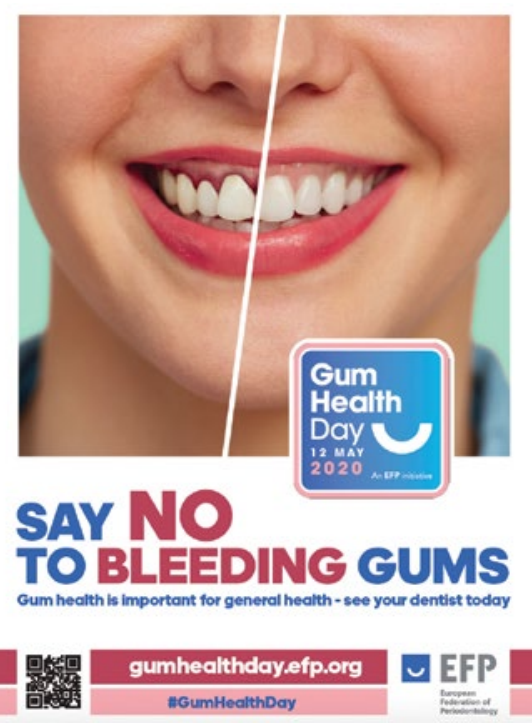

More than 40 countries joined Gum Health Day 2020 - from Europe, the Americas, Africa, Middle East, Asia, and
Australasia. The European Federation of Periodontology (EFP) and its affiliated societies organised a wide variety of initiatives - most of them digital because of the COVID-19 pandemic - which include educational videos, publications, social-media campaigns, an Instagram Live session, and other online initiatives.

In the framework of Gum Health Day 2020, the EFP encourages dentists, researchers, and other health-related professionals to sign and disseminate the EFP Manifesto: Perio and General Health, an international call to action for the prevention, early detection, and treatment of gum disease. Individuals and organisations are invited to endorse it by accessing http://www.efp.org/efpmanifesto/ and join the more than 1,100 professionals, dental practices, companies, and universities who have supported it so far. 